\title{
Detection of Aujeszky's disease using blood samples collected on Whatman paper disks
}

\begin{abstract}
M. ELOIT, B. TOMA
Ecole Nationale Vétérinaire d'Alfort, Chaire des maladies contagieuses, 94704 Maisons-Alfort Cedex

A commercialized enzyme-linked immunosorbent assay (ELISA) was used to detect antibodies to Aujeszky's disease in 525 blood samples collected on Whatman paper disks. A sensitivity of $98 \%$ and a specificity of $100 \%$ were obtained compared to an ELISA applied to individual serum samples. Parallel to that, 200 groups of 5 disks including at most one disk impregnated with blood from a seropositive pig were analysed. This led to modify the level of positiveness recommended by the producer to adapt it to the study of disk groups. In these conditions, a sensitivity of $97 \%$ and a specificity of $100 \%$ were obtained compared to the analysis of individual sera.
\end{abstract}

\section{Utilization in the practice of an experimental vaccine against atrophic rhinitis in the pig: effect on nasal lesions and performance}

\author{
Marylène $\mathrm{KOBISCH}^{(1)}$, P. MORVAN ${ }^{(2)}$
}

(1) Ministère de l'Agriculture, Direction de la Qualité, Services Vétérinaires, Station de Pathologie Porcine, B.P. 9, Les Croix, 22440 Ploufragan

(2) Laboratoire Départemental d'Analyses des Côtes-du-Nord, B.P. 14, 8, place du 74 R.I., 22021 Saint-Brieuc.

The efficiency of an experimental vaccine including Bordetella bronchiseptica and Pasteurella multocida (capsular group D) was tested in two herds exhibiting clinical signs of atrophic rhinitis. Administration of the vaccine to pregnant sow induced seral antibodies transmitted to piglets via the colostrum. The sanitary status of the herd was found to affect the efficiency of the vaccine. Indeed, in the first herd (good sanitary status), vaccination reduced by $60 \%$ the number of pigs with nasal lesions, whereas in the second one (where most parameters were negative), the efficiency was less marked since the number of pigs with turbinate atrophy was only reduced by $19 \%$. However, in both cases, vaccination reduced the extent of turbinate damage. The vaccination did not affect either the performance or the pulmonary lesions which concerned a high proportion of animals.

\section{IV. - FEEDING}

\section{Energy utilization and energy requirements in pregnant and lactating sows}

\section{J. NOBLET, M. ETIENNE}

INRA, Station de Recherches Porcines, Saint-Gilles, 35590 L'Hermitage.

Energy and nitrogen balances were carried out in respiration chambers in 32 pregnant gilts ( 90 balances) and 20 lactating sows (lactation length : 21 days, 40 balances). During pregnancy, animals were fed 6.6 (24 gilts) or 8.0 ( 8 gilts) Mcal ME per day. During lactation, mean ME 